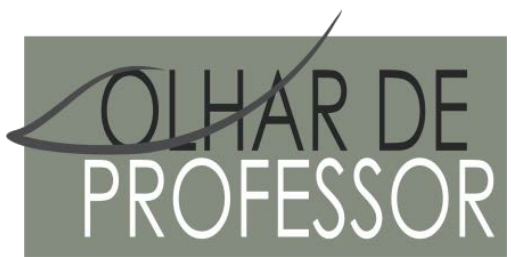

DOI: 10.5212/OLHARPROFR.v.21i1.0007

\title{
DIAGNÓSTICO DE LOS PROCESOS PEDAGÓGICOS LLEVADOS A CABO EN EL LABORATORIO DE FÍSICA REFERENTES A LA ELECTRICIDAD EN EL CONTEXTO UNIVERSITARIO
}

\section{DIAGNOSIS OF PEDAGOGICAL PROCESSES CARRIED OUT IN THE PHISICS LABORATORY REGARDING TO ELECTRICITY IN THE UNIVERSITY CONTEXT \\ DIAGNÓSTICO DE PROCESSOS PEDAGÓGICOS REALIZADOS NO LABORATÓRIO DE FISICA REFERENTE 0 ELETRICIDADE NO CONTEXTO DA UNIVERSIDADE}

\author{
RICHAR NICOLÁS DURÁN \\ JESÚS RAMÓN BRICEÑO ** \\ IRIS NAILE MATERAN ${ }^{* * *}$ \\ FRANK DABOÍN ${ }^{* k *}$
}

\begin{abstract}
Resumen: El trabajo de investigación que se presenta tuvo como objetivo central Diagnosticar los procesos pedagógicos llevados a cabo por los docentes en la planificación de la asignatura de Laboratorio de Física en los tópicos relacionados con la Electricidad. Esto conlleva a una idea secundaria que es revisar cómo son estudiados los conceptos junto con diversas teorías fundamentadas en la Electricidad y cómo éstas son implementadas en la Educación Universitaria. La investigación se abordó siguiendo la metodología de proyecto descriptivo, junto con estudio de campo y documental, lo que permitió hacer dicho diagnóstico. La población de estudio estuvo conformada por cinco (05) profesores del área de Física en una Institución Universitaria del estado Trujillo. La técnica aplicada fue una entrevista semi-estructurada con (09) nueve preguntas abiertas, que sirvió para la verificación del abordaje durante las clases de Laboratorio de Física. Cabe mencionar que se muestra los resultados y análisis más resaltantes de la investigación, donde arrojó diversas perspectivas de abordar un curso de laboratorio siguiendo ciertos parámetros ya predeterminados sin tomar en cuenta la evolución en cuanto a herramientas didácticas y tecnológicas. Para dichos resultados se utilizó la herramienta Tecnológica ATLAS.ti que es para realizar análisis de datos cualitativos.
\end{abstract}

Palabras claves: Diagnóstico, electricidad, estrategias didácticas, enseñanza-aprendizaje, teoría constructivista.

Abstract: The main objective of the research work was to diagnose the pedagogical processes carried out by the teachers in the planning of the theme of the Physics Laboratory in the subjects related to Electricity. This leads to

\footnotetext{
* Licenciado en Educación Mención Física y Matemática de la Universidad de Los Andes. Estado Mérida-Venezuela. Investigador adscrito al Grupo de Investigación en Ciencia su enseñanza y Filosofía (GRINCEF) ULA-NURR E-mail: rduran.ula@gmail.com

*** Profesor Titular de la Universidad de Los Andes, en el área de Física. Adscrito al Grupo de Investigación en Ciencia su Enseñanza y Filosofía GRINCEF. E-mail: jesusrbb@gmail.com

${ }^{* * *}$ Profesora Asociado de la Universidad de Los Andes, en el área de Física. Adscrita al Grupo de Investigación en Ciencia su Enseñanza y Filosofía GRINCEF. E-mail: irisnaile@gmail.com

***** Profesor Asociado de la Universidad de Los Andes, en el área de Física. Adscrito al Grupo de Investigación en Ciencia su Enseñanza y Filosofía GRINCEF. E-mail: fisicachess@gmail.com
} 
a secondary idea which is to review how concepts are studied in conjunction with various electricity-based theories and how they are implemented in university education. The research was approached following the descriptive methodology of the project, along with the field and documentary study, which allowed to make this diagnosis. The study population consisted of five (05) professors from the Physics area of a University Institution in the state of Trujillo. The applied technique was a semi-structured interview with (09) nine open questions, which served to verify the approach during the classes of the Laboratory of Physics. It is worth mentioning that it presents the results and most important analyzes of the research, where it presented several perspectives to approach a laboratory course following predetermined parameters, without taking into account the evolution in terms of didactic and technological tools. For these results, the technological tool ATLAS.ti was used, which is the qualitative analysis of the data.

Key words: Diagnosis, electricity, didactic strategies, teaching-learning, constructivist theory.

Resumo: $O$ trabalho de pesquisa que se apresenta teve como objetivo central diagnosticar os procesos pedagógicos desenvueltos pelos profesores no planejamento na disciplina de Laboratório de Física nos tópicos relacionados com eletricidade. Isto implica revisar como são estudados os conceitos junto com diversas teorías fundamentadas na eletricidade e como estão são implementadas na educação universitária. A pesquisa se desenvolveu seguindo a metodología de projeto descritivo e envolveu estudo de campo e documental, o que permitiu fazer este diagnóstico. A população estudada foram (05) profesores da área de física numa instituição universitária do estado de Trujillo. A técnica utilizada foi a entrevista semi-estruturada com (09) nove perguntas abertas voltadas a verificar a abordagem durante as aulas do Laboratório de Física. Vale ressaltar que se traz os principais resultados e análises da pesquisa, apresentando diversas perspectivas para a abordagem de um laboratorio seguindo certos parámetros já predeterminados sem levar em conta a evolução de ferramentas didáticas e tecnológicas. Para estes resultados utilizou-se a ferramenta tecnológica ATLAS.ti de análise qualitativa dos dados.

Palavras-chave: Diagnóstico. Eletricidade. Estratégias didáticas. Ensino-aprendizagem. Teoria construtivista.

\section{INTRODUCCIÓN}

En el presente artículo se describe un análisis de cómo está siendo implementada las prácticas de laboratorio de Física General, haciendo énfasis en el contenido de electricidad ya que es un tema interesante de estudiar y que los estudiantes que estudian tanto la carrera de educación mención Física como en la rama de la Ingeniería deben tener conocimientos bien claros para abordar cualquier problema de la vida cotidiana. Esto se realizó haciendo una entrevista semi-estructurada a los profesores que imparten dicha asignatura, en una Universidad del estado Trujillo, Venezuela.

La Física como ciencia que estudia la naturaleza a lo largo del tiempo ha sido usada para tantos fines transcendiendo sus fronteras, que hoy día la modernización de la sociedad es gracias a sus avances. Un estudiante con buen rendimiento académico en Física, Química o Biología es considerado como una persona capaz de desenvolverse profesionalmente en el área de las ciencias ejecutando investigación o producción, dependiendo de sus habilidades y destrezas, la mayoría de los jóvenes de nuestras casas de estudio a nivel superior enfrentan un gran temor de cómo abordar el aprendizaje eficaz, además del tabú perdurable en la cotidianidad de que las ciencias son una de las asignaturas más difíciles.

Se debe considerar que la Física es muy rica en matices didácticos, y que para la comprensión del mundo moderno en el siglo XXI desarrollado tecnológicamente, es necesario tener conocimientos sólidos respecto a la Física. Debe el estudiante llevarse la idea que todo país que quiera mantenerse en los primeros lugares, con industrias competitivas, ha de potenciar el nivel de calidad en la enseñanza de las ciencias en todos los niveles, sin que esto a de interpretarse como el abandono o desprecio de la formación humanística absolutamente necesaria para crear ciudadanos libres y socialmente responsable 
La Física Clásica basada en la mecánica Newtoniana, leyes de Maxwell del electromagnetismo, entre otros, se adapta perfectamente al análisis del comportamiento de los objetos macroscópicos en función de variables que se pensaban que eran controlables y medibles, como posición, velocidad, aceleración, energía, entre otras. Pero una vez que nos introducimos en el mundo atómico, nos encontramos en presencia de nuevos fenómenos que requieren el estudio de nuevos conceptos para su análisis y descripción, de tal manera que podamos explicar a los estudiantes temas que suelen ser un poco abstractos para ser semejanza con el mundo real que sin embargo se puede hacer un buen uso estratégico-didáctico para su mejor comprensión.

Cabe destacar, que antes de abordar y conocer un tema nuevo, es fundamental el estudio de conceptos en la mecánica clásica propuesta por Isaac Newton, partiendo de las nociones del alumno respetando su lugar, espacio e interés, y además estimulando a despertar las ganas de conocer el mundo de la Física. Debe señalarse que, es de gran importancia el implemento de los laboratorios en la enseñanza de ciencias. Es por ello que el trabajo práctico en el laboratorio proporciona al estudiante la experimentación y el descubrimiento personal por sí mismos, de manera que los conocimientos adquiridos en conceptos y definiciones van a ser mejor asimilados por los mismos, en otras palabras, el estudiante no solo debe conocer, simular y recrear el experimento, si no que a partir de los datos verifique las leyes que rigen el fenómeno.

Para Barolli, Laburú e Guridi (2010, p. 105), expresa que existe "un cierto desfasaje en la producción de trabajos centrado en el debate teórico sobre el papel de los laboratorios y la producción de conocimientos utilizados por los estudiantes en la resolución de problemas específicos de los trabajos prácticos.” De manera que, no podemos ver por separada la parte teórica junto con la práctica. De manera tal que se debe tomar conciencia sabiendo que los estudiantes traen consigo conocimientos previos, sin embargo se les debe facilitar material como textos y guías teóricas, para que así sea más factible la asimilación concreta de nuevos conocimientos que sin duda alguna conllevaría a la adquisición de mayores destrezas al momento de ejecutar una demostración experimental.

Para Arruda (2003), la didáctica de la Física desarrollada bajo el principio dialéctico de la unidad teoría-práctica, ejecuta funciones de carácter educativo y científico al mismo tiempo. La unidad correcta de esa relación exige un cambio de paradigma de la práctica pedagógica, es decir, cambiar el paradigma de la Pedagogía Tradicional, mecánico-cartesiano; modelo del alumno - tabula rasa, para el paradigma Histórico-Cultural; modelo del alumno activo, buscando garantizar actividades que proporcionan el desarrollo del conocimiento científico, la adquisición de habilidades y hábitos. En este sentido, se hace referencia que el nivel de enseñanza de la Física en el siglo XXI podría ser mejor aprovechada por parte de los estudiantes si se establecen mecanismos didácticos que permitan desarrollar en un ambiente educativo propicio para el mejoramiento académico. Mostrando herramientas que permitan al alumno desarrollar el interés por el conocimiento de la Física dando explicación desde su origen, es decir, incentivar a la investigación de esos fenómenos naturales y la gran variedad de aplicabilidad con el entorno.

\section{LA ACTIVIDAD EXPERIMENTAL EN FISICA.}

Para efectos de la presente investigación se entiende como actividad experimental aquella actividad educativa en ciencias que para su realización incluye una experiencia que sea real, efectuada por el educando o por el maestro con la colaboración de los estudiantes, empleando materiales de su entorno, y que dirija y articule el proceso de enseñanza-aprendizaje y evaluación de algún concepto científico.

De acuerdo con esta concepción de actividad experimental y teniendo en cuenta que la experimentación permite el contacto directo con los fenómenos, se hace importante analizar cuáles son las implicaciones de este tipo de actividades en el proceso de enseñanza-aprendizaje y evaluación de las ciencias naturales. En consecuencia, en el camino de construir los modelos teóricos escolares, las 
actividades experimentales juegan un papel muy importante ya que su realización implica la producción de procesos cognitivo lingüísticos sustentados por el lenguaje en todas sus modalidades; hecho que contribuye a que poco a poco, y con ayuda de los especialistas en ciencias, las maneras de pensar y representar de los estudiantes se vayan acercando a las reglas de juego de la ciencia esto según Izquierdo, Sanmartí y Espinet (1999).

\title{
METODOLOGÍA
}

En referencia a lo planteado, esta investigación es un proyecto de tipo predictiva, porque consiste en diagnosticar por medio de una entrevista, el cual permitió llevar a cabo los objetivos propuestos, el universo de estudio fueron un grupo de docentes que imparten dicha asignatura, en la Universidad de Los Andes Núcleo Universitario Rafael Rangel.

En este sentido la Universidad Pedagógica Experimental Libertador (UPEL) define el proyecto factible de la siguiente manera:

\begin{abstract}
El proyecto descriptivo consiste en la investigación, elaboración y desarrollo de una propuesta de un modelo operativo viable para solucionar diagnosticar algún problema, requerimientos o necesidades de organizaciones o grupos sociales; puede referirse a la formulación de políticas, programas tecnologías, métodos o procesos. El proyecto debe tener apoyo en una investigación de tipo documental, de campo o diseño que incluya ambas modalidades. (UPEL, 2012, p. 13, apud FUENTES; RIVERO; PÉREZ, 2016, p. 23).
\end{abstract}

De tal manera, que para esta investigación se seleccionóel grupo de docentes, el cual fueron un total de (05) cinco, y que además hayan dictado la asignatura de Laboratorio de Física General en los semestres A-2017 al B-2018 respectivamente. Posteriormente, para la validación la muestra, estuvo conformada por revisión de expertos, específicamente en el área de física los cuales están adscritos al Grupo de Investigación en Ciencia su Enseñanza y Filosofía (GRINCEF). Observe:

Cuadro No 1 Cuadro de abordaje de la investigación.

\begin{tabular}{|l|l|}
\hline Fase & Etaas \\
\hline Documental & $\begin{array}{l}\text { 1. Revisión Teórica. } \\
\text { 2. Consulta con los expertos en el área de Física. } \\
\text { 3. Selección de los sujetos de investigación }\end{array}$ \\
\hline Ejecución & $\begin{array}{l}\text { 4. Entrevista semi-estructurada a cinco (5) profesores que han dictado los } \\
\text { cursos de laboratorio de física en el NURR. }\end{array}$ \\
\hline Categorización & $\begin{array}{l}\text { 5. La entrevista a los profesores se contextualizó, para poder determinar los } \\
\text { invariantes operatorios, reglas de acción y anticipaciones, se aplicó el } \\
\text { programa ATLAS.ti. }\end{array}$ \\
\hline Interpretación & $\begin{array}{l}\text { 6. Interpretar los resultados estadísticos arrojados del diagnóstico al igual } \\
\text { que las invariantes operatorias, reglas de acción y anticipaciones para } \\
\text { representar las respuestas de los entrevistados (informantes clave). }\end{array}$ \\
\hline Conclusiones & 7. Respuesta a la pregunta de la investigación \\
\hline
\end{tabular}

Fuente: Autor.

Luego se empleó el instrumento seleccionado, el cual consistió de una entrevista semiestructurada con preguntas abiertas, realizada a profesores que imparten el curso de Laboratorio de Física General. 
Dicho instrumento contó con (09) ítem de preguntas abiertas, éstas tenían la finalidad de verificar como se han desarrollado las clases de laboratorio de Física durante los distintos semestres desde A-2017 al B-2018 y lograr apreciar hasta qué punto son productivas las aplicaciones de las practicas diseñadas por el área de física y como es el desempeño de los estudiantes.

En esta investigación se utilizó como referencia el programa del curso de Laboratorio de Física propuesto por el departamento de Física y Matemática del NURR, y distintas fuentes donde muestra los experimentos ya realizados durante diversos cursos ya previstos.

\section{ANÁLISIS E INTERPRETACIÓN DE LOS RESULTADOS OBTENIDOS EN LAS ENTREVISTAS.}

Posteriormente para el tratamiento y análisis de los datos en la entrevista a los cinco (5) profesores, se empleó el uso del Software Atlas.ti, cuyo programa no tiene como finalidad automatizar el proceso de análisis, al contrario permite ayudar al investigador agilizando considerablemente muchas de las actividades implicadas en el análisis cualitativo y la interpretación, como por ejemplo la segmentación del texto en pasajes o citas, la codificación, o la escritura de comentarios y anotaciones; es decir, todas aquellas actividades que, de no disponer del programa, se tendrían que realizar ayudándonos de otras herramientas como papel, lápices de colores, tijeras, fichas, fotocopias... (MUÑOZ, 2005, p. 2).

Para poder analizar los datos en el Software, fue necesario importar todas las respuestas de los ítems transcritas en formato RF a la Unidad Hermenéutica (denominación del archivo donde se almacena todo el trabajo) como documentos primarios: "datos brutos” que son la base del análisis. Luego se procedió a la selección y asignación de códigos CODES al contenido del instrumento, en esta tarea, seguí un procedimiento deductivo-inductivo, ya que comencé por la creación de códigos libres extraídos de la propia formulación teórica del estudio y de las dimensiones directamente relacionadas con las preguntas de investigación.

Cuadro No 2 Códigos o características.

\begin{tabular}{|ll|l|}
\hline \multicolumn{2}{|c|}{ Códigos Libres } & \\
\hline 1. & Personalidad del Docente. & $5 . \quad$ Relación profesor-alumno \\
2. & Características a desarrollar en clases. & $6 . \quad$ Actualización de las clases en el laboratorio \\
3. & Estructura de clases en el laboratorio. & $7 . \quad$ Valoración de los estudiantes \\
4. & Condiciones de enseñanza-aprendizaje en el & 8. Prácticas de electricidad \\
& laboratorio. & 9. Visión de objetivos propuestos en cada práctica. \\
& & 10. Aportes de las prácticas en lo profesional. \\
\hline
\end{tabular}

Fuente: Autor.

De los análisis de las entrevistas realizadas a los profesores que han tenido la oportunidad de dictar cursos de Laboratorio de Física General fueron esquematizados por medio de la codificación que se le asignó a través de la herramienta Atlas ti. Seguidamente se muestra los (02) dos primeros encuestados como observaremos a continuación.

Informante clave A

\begin{tabular}{|l|l|}
\hline \multicolumn{2}{|l|}{ Ficha de identificación } \\
\hline Título: Lic en Educ. Física y Matemática & Sexo: Masculino \\
\hline Experiencia docente: 4 años & Edad: 30 \\
\hline
\end{tabular}


Figura $N^{\circ} 1$. Representación de los invariantes operatorios, anticipaciones y reglas de acción del informante clave A.

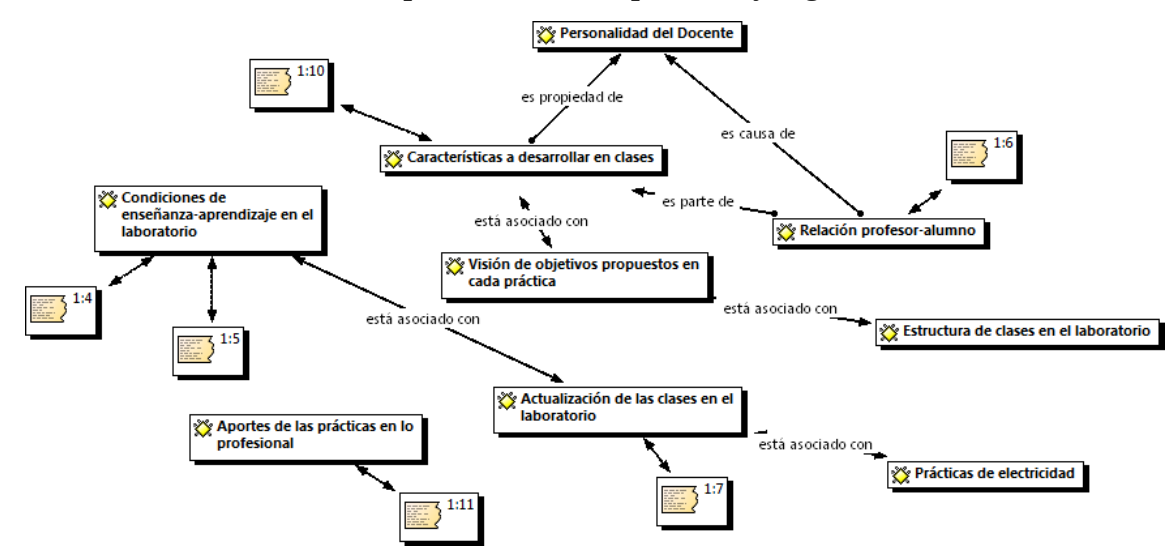

Citas del abordaje escrito del Caso A

(1:4)...motivación del estudiante respecto a experimentar en el tema...

(1:5)...uso de TIC con simuladores para que obtengan tentativamente las posibles correlaciones y leyes que se van a discutir...

(1:6)...horizontal fundamentada en las clases expositivas y cambios conceptuales...

(1:7)...guías actualizadas y ajustadas a los materiales existentes en el laboratorio...

Realizando el respectivo análisis de respuestas en el caso A, se pudo constatar que según los códigos más resaltantes surge el hecho de tomar en consideración el análisis detallado de los textos bibliográficos que se les asigna al estudiante, en base a esto el docente enfoca sus estrategias, primero en indagar que conocimientos básicos trae consigo el estudiante para poder afianzarlos con mayor fluidez profundizar en ellos, de manera tal que, tenga las destrezas y competencias al momento de enfrentarse con la experimentación más aun en la parte de electricidad. El docente en cuestión tiene grandes expectativos respecto a cómo darle una evolución a la experimentación por medio de las TIC y proyectar el uso de programas llamados simuladores, en particular cuando se trata de las prácticas de electricidad puesto que ocurren fenómenos que suelen ser algo abstractos y formales donde el estudiante no lo visualiza muy atractivo.

\section{Informante clave B}

\begin{tabular}{|l|l|}
\hline Título: Lic en Educ. Física y Matemática & Sexo: Femenino \\
\hline Experiencia docente: 10 años & Edad: 34 \\
\hline
\end{tabular}

Figura $\mathrm{N}^{0}$ 2. Representación de los invariantes operatorios, anticipaciones y reglas de acción del Caso B

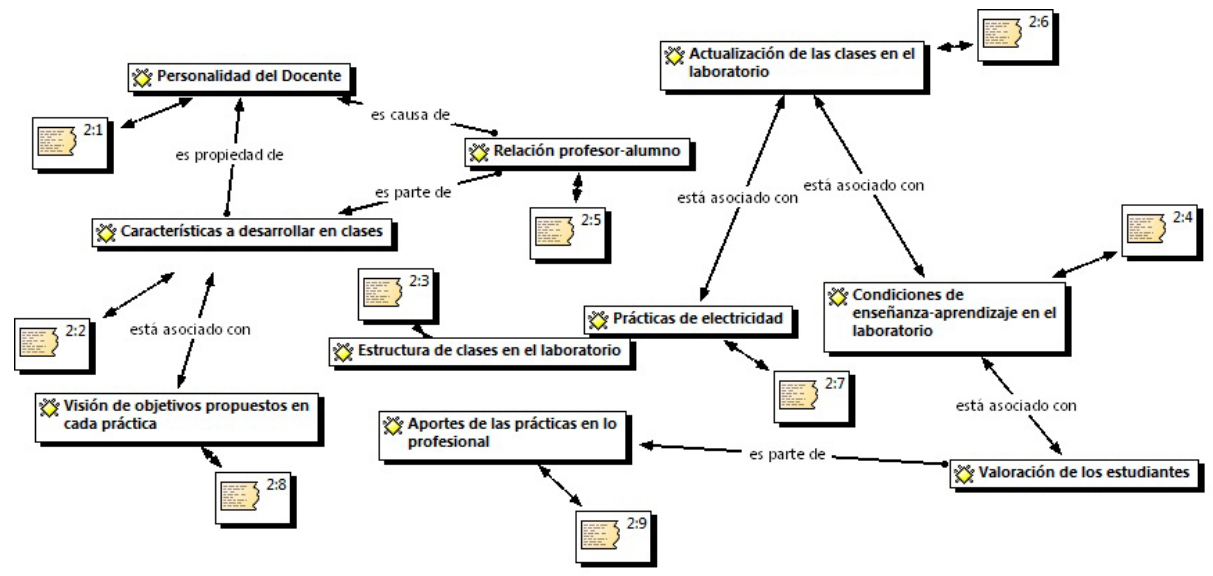




\section{Citas del abordaje escrito del Caso B}

(2:1)... un docente social que trata de buscar el equilibrio en la formación de los estudiantes... (2:2)...el estudiante debe construir su propio aprendizaje a través de la experiencia, el estudio previo y la debida preparación ...

(2:3)...organizo las clases de manera tal que los estudiantes sean los actores principales del aprendizaje...

(2:4)...un ambiente donde el trabajo en conjunto y la preparación de cada uno de los miembros del grupo de poner en manifiesto...

(2:6)...restaurando las actividades de laboratorio de manera consensuada con los otros miembros del área...

(2:7)... realizando las actividades por sí mismos, dejando atrás el temor que puede producir una descarga eléctrica...

(2:8)...encuentra en la sesiones de laboratorio una oportunidad para la construcción de conocimiento...

Para el caso del informante clave B, este fue bastante explícito en sus respuestas, da a entender que es un docente que trata de buscar el equilibrio en los estudiantes durante su formación (1:1), lo cual es necesario al momento de trabajar en el aula de clases y más aun con un curso de laboratorio de Física, se ve que este docente da oportunidad para que el estudiante construya su propio aprendizaje, algo que en el ámbito educativo siempre se trata de alcanzar, también puntualiza que para evaluar las condiciones del laboratorio se debe realizar en consenso con los demás profesores del área (2:6), también señala que es de iniciativa permitir que el estudiante rompa esa barrera de "miedo" al enfrentarse con los equipos de electricidad, esto es un punto sumamente importante, puesto que hay que brindarle al estudiante esa seguridad y confianza al momento de realizar algún montaje experimental.

Todo lleva a una relación entre las partes, y los elementos que la integran, los cuales deben articularse de manera holística conformando un todo, eso se aprecia en el gráfico (esquema) anterior, lo que trae como conclusión que una vez cumplida todas esas condiciones que se deben desarrollar en los cursos de laboratorio de Física, se tendría un mejor provecho tanto para para el estudiante como para el profesor.

\section{CONSIDERACIONES FINALES}

La investigación se trató de diagnosticar las necesidades y condiciones de enseñanzaaprendizaje de la electricidad, donde por medio del desarrollo se alcanzó evidenciar cómo es el desarrollo de las clases en los dos cursos de laboratorio de Física específicamente en las prácticas de electricidad, además de realizar una entrevista a los profesores que imparten dicha asignatura, la investigación se abordó en la carrera de Educación mención Física y Matemática, usando como referente teórico la Teoría constructivista, en una primera instancia las situaciones que dan sentido a los conceptos parten de la adquisición de conocimientos y como es moldeada por las situaciones previamente dominadas, en tanto que el progresivo dominio de un concepto se logra al enfrentar una amplia variedad de situaciones y considerar al estudiante como un sistema dinámico de construcción de esquemas de asimilación, los cuales pueden entrar en conflicto, además de generar procesos de acomodación y de construcción de nuevos esquemas de asimilación.

La física debe estar rodeada del complemento práctico que facilite un mejor aprendizaje, la manera con la que se presenta, queda de parte del docente hacer uso de una infinidad de herramientas y estrategias que permiten un mejor desenvolvimiento. Es importante que el docente actual este en contante revisión de las nuevas teorías y formas de abordar en la enseñanza. De manera que la 
educación prevalezca la calidad y no en la cantidad, que esto a su vez se siga fortaleciendo con las tendencias actuales. A los docentes que imparten el área de ciencias, en este caso la Física, es importarte que esté en constante cambio, es decir; que sea innovador, investigador y planificador. Esto conlleva a que en él se generen modelos que permitan una mejor enseñanza, y que sin duda hagan un docente productivo para sus estudiantes, dejando en ellos técnicas que les permitan generar conocimientos reflexivos sobre hechos que más que, Físicos son aspectos que se manifiestan en la vida cotidiana y que forman parte del quehacer rutinario de cada uno de nosotros, en nuestra interacción con todos los demás elementos del cosmos con los cuales compartimos nuestra existência.

\section{REFERENCIAS}

ARRUDA, J. R. C. Un modelo didáctico para enseñanza aprendizaje de la física. Revista Brasileira de Ensino de Física, [s. l.], v. 25, n. 1, p. 86-104, mar. 2003. Disponível em: http://www.scielo.br/pdf/rbef/v25n1/a11v25n1.pdf Acesso em: 18 dez. 2014.

BAROLLI, E.; LABURÚ, C. E.; GURIDI, V. M. Laboratorio didáctico de ciencias: caminos de investigación. Revista Electrónica de Enseñanza de las Ciencias, [s. l.], v. 9, n. 1, p. 88-110, jan./abr. 2010.

FUENTES, W.; RIVERO, E. J.; PÉREZ, V. Manual de trabajo para el estudio de trabajo y energía (energía cinética y potencial). Bogotá: Universidad de los Andes, 2016. 42 p.

IZQUIERDO, M.; SANMARTI, N.; SPINET, M. Fundamentación y diseño de las prácticas escolares en ciencias experimentales. Enseñanza de las ciencias, [s. l.], v. 17, n.1, p. 45-59, jan./abr. 1999.

MUÑOZ, J. Análisis cualitativo de datos textuales con ATLAS.ti 5.0. Barcelona: Universitat Autònoma de Barcelona, 2005. 